\title{
Stability Result for a Kirchhoff Beam Equation with Variable Exponent and Time Delay
}

\author{
Jorge Ferreira $^{1}$, Erhan Pişkin ${ }^{2}$, Carlos Raposo $^{3}$, Mohammad Shahrouzi $^{4 *}$ and Hazal Yüksekkaya ${ }^{2}$ \\ ${ }^{1}$ Federal Fluminense University, Department of Exact Sciences, Rio de Janeiro, Brazil \\ ${ }^{2}$ Dicle University, Department of Mathematics, Diyarbakir, Turkey \\ ${ }^{3}$ Department of Mathematics, Federal University of São João del-Rei, Minas Gerais, Brazil \\ ${ }^{4}$ Department of Mathematics, Jahrom University, Jahrom, P.O.Box: 74137-66171, Iran \\ *Corresponding author
}

\section{Article Info}

Keywords: Kirchhoff beam equation, stability, time delay, variable exponents. 2010 AMS: 35B35, 35B40,35LO5.

Received: 25 November 2021

Accepted: 22 January 2022

Available online: 07 March 2022

\begin{abstract}
This paper is concerned with a stability result for a Kirchhoff beam equation with variable exponents and time delay. The exponential and polynomial stability results are proved based on Komornik's inequality.
\end{abstract}

\section{Introduction}

Let $\Omega \subset \mathbb{R}^{n}$ be a bounded domain with sufficiently smooth boundary $\partial \Omega$. For $(x, t) \in \Omega \times \mathbb{R}^{+}$we consider a Kirchhoff beam equation with variable exponents and time delay given by

$$
u_{t t}+\Delta^{2} u-M\left(\|\nabla u\|^{2}\right) \Delta u+\mu_{1} u_{t}(x, t)\left|u_{t}\right|^{m(x)-2}(x, t)+\mu_{2} u_{t}(x, t-\tau)\left|u_{t}\right|^{m(x)-2}(x, t-\tau)=0,
$$

with Dirichlet boundary condition

$$
u(x, t)=0 \text { in } \partial \Omega \times[0, \infty),
$$

and initial data

$$
\begin{array}{r}
u(x, 0)=u_{0}(x) \text { in } \Omega, \\
u_{t}(x, 0)=u_{1}(x) \text { in } \Omega, \\
u_{t}(x, t-\tau)=f_{0}(x, t-\tau) \text { in } \Omega \times(0, \tau),
\end{array}
$$

where $\tau>0$ is time delay term, $\mu_{1}$ is a positive constant, $\mu_{2}$ is a real number. $M(s)$ is a positive $C^{1}$-function like $M(s)=a+b s^{\gamma}$ for $s \geq 0$, specially we take $a, b=1$ and $\gamma>0$. The functions $u_{0}, u_{1}, f_{0}$ are the initial data to be specified later.

The variable exponent $m(\cdot)$ is a measurable function on $\bar{\Omega}$ satisfying

$$
2 \leq m^{-} \leq m(x) \leq m^{+} \leq m^{*}, \text { where } m^{-}=e s s \inf _{x \in \Omega}(x), m^{+}=e s s \sup _{x \in \Omega} m(x) \text { e } m^{*}=\frac{2(n-2)}{n-4} \text { if } n \geq 5 .
$$

The problems with variable exponents arise in many branches in sciences such as nonlinear elasticity theory, electrorheological fluids and image processing $[7,8,26]$. Time delay often appears in many practical problems such as thermal, economic phenomena, biological, chemical 
and physical [12].

One of the first mathematical analysis of beam equation for $\Omega=(0, L) \subset \mathbb{R}, L>0$,

$$
u_{t t}+u_{x x x x}-M\left(\int_{0}^{L}\left|u_{x}\right|^{2} d x\right) u_{x x}=0
$$

was done by Ball (1973) [6]. Tucsnak (1996) [29] extended (1.7) for the beam equation in $\Omega \subset \mathbb{R}^{n}$

$$
u_{t t}+\Delta^{2} u+M\left(\|\nabla u\|^{2}\right)(-\Delta u)=0,
$$

where

$$
\|\nabla u\|^{2}=: \int_{\Omega}|\nabla u|^{2} d x .
$$

The problem (1.8) is called of Kirchhoff type in reason of the one-dimensional nonlinear equation (1.9) proposed by Kirchhoff [13] (1883),

$$
\frac{\partial^{2} u}{\partial t^{2}}-\left(\frac{\tau_{0}}{m}+\frac{k}{2 m L} \int_{0}^{L}\left(\frac{\partial u}{\partial x}\right)^{2} d x\right) \frac{\partial^{2} u}{\partial x^{2}}=0,
$$

where $\tau_{0}$ is the initial tension, $m$ the mass of the string and $k$ the Young's modulus of the material of the string. This model, in connection with some problems in nonlinear elasticity, describes small vibrations of a stretched string of the length $L$ when only the transverse component of the tension is considered.

This kind of problem (1.9) is obtained from the model (1.10), first proposed by Woinowsky-Krieger [30] (1950), for the transverse motion of an extensible beam of the length $L$ whose ends are attached at a fixed distance

$$
\frac{\partial^{2} u}{\partial t^{2}}+\frac{E I}{\rho} \frac{\partial^{4} u}{\partial x^{4}}+\left(\frac{H}{\rho}+\frac{E A}{2 \rho L} \int_{0}^{L}\left|\frac{\partial u}{\partial x}\right|^{2} d x\right)\left(-\frac{\partial^{2} u}{\partial x^{2}}\right)=0
$$

where $L, E, I, \rho, H$ and $A$ denote, respectively, the length of the beam in the rest position, the Young's modulus, the cross-sectional moment of inertia, the mass density, the tension in the rest position, and the cross-sectional area.

Antontsev et. al. [3], considered the nonlinear plate (or beam) Petrovsky equation with variable exponents as follows:

$$
u_{t t}+\Delta^{2} u-\Delta u_{t}+\left|u_{t}\right|^{p(x)-2} u_{t}=|u|^{q(x)-2} u .
$$

The authors, by using the Banach contraction mapping principle, obtained the local weak solutions. Also, they showed that the solution is global if $p(\cdot) \geq q(\cdot)$. Moreover, the authors proved that a solution with negative initial energy and $p(\cdot)<q(\cdot)$ blows up in finite time. In [4], Antontsev et al., considered the Timoshenko-type equation with variable exponents as follows:

$$
u_{t t}+\Delta^{2} u-M\left(\|\nabla u\|^{2}\right) \Delta u+\left|u_{t}\right|^{p(x)-2} u_{t}=|u|^{q(x)-2} u .
$$

The authors proved the local existence of the solution. Moreover, they investigated the nonexistence of solutions for negative initial energy. In [5], Antontsev et al., studied the nonlinear $p(x)$-Laplacian equation with time delay and variable exponents as follows:

$$
u_{t t}-\Delta_{p(x)} u+\mu_{1} u_{t}(x, t)\left|u_{t}\right|^{m(x)-2}(x, t)+\mu_{2} u_{t}(x, t-\tau)\left|u_{t}\right|^{m(x)-2}(x, t-\tau)=b u|u|^{q(x)-2} .
$$

The authors proved the blow up of solutions. Then, by applying an integral inequality due to Komornik, they obtained the decay result. There are few results on Kirchhoff beam equation with delay. In [10] was considered the following nonlinear viscoelastic Kirchhoff beam equation with a time delay term in the internal feedback, given by

$$
u_{t t}+\Delta^{2} u-\operatorname{div} F(\nabla u)-\sigma(t) \int_{0}^{t} g(t-s) \Delta^{2} u(s) d s+\mu_{1} u_{t}\left|u_{t}\right|^{m-1}(x, t)+\mu_{2} u_{t}\left|u_{t}\right|^{m-1}(x, t-\tau)=0
$$

where $\Omega \subset \mathbb{R}^{n},(n \geq 1)$ is a bounded domain with smooth boundary $\partial \Omega$. The function $u=u(x, t)$ is the transverse displacement, and $\sigma(t)$ and $g(t)$ are positive functions defined on $\mathbb{R}^{+} . \mu_{1}, \mu_{2}$ are positive constants and $\tau>0$ represents the time delay. Under suitable assumptions, the authors established the general rates of energy decay by using the energy perturbation method.

Kafini and Messaoudi [16], studied the equation with variable exponents and delay term as follows:

$$
u_{t t}-\Delta u+\mu_{1} u_{t}(x, t)\left|u_{t}\right|^{m(x)-2}(x, t)+\mu_{2} u_{t}(x, t-\tau)\left|u_{t}\right|^{m(x)-2}(x, t-\tau)=b u|u|^{p(x)-2} .
$$

They established the decay estimates and global nonexistence results for the equation (1.14).

Santos et al. [27], investigated the existence and the decay of the beam equation as follows:

$$
u_{t t}+\Delta^{2} u-M\left(\|\nabla u\|^{2}\right) \Delta u-\int_{0}^{t} h(t-s) \Delta u(s) d s+\alpha u_{t}=0,
$$

in a non-cylindrical domain. Recently, some other authors investigate hyperbolic type equations (see [11,21-25, 28]).

Our aim in this work is to prove the stability of solutions for the Kirchhoff beam equation with the delay term $\left(\mu_{2} u_{t}(x, t-\tau)\right)$ and variable exponents which make the problem more different than from those considered in the literature. This manuscript extends the result of [16] to Kirchhoff beam equation.

The paper is organized as follows: In Section 2, the definition of the variable exponents Sobolev and Lebesgue spaces are stated. In Section 3 , we obtain the stability result. 


\section{Preliminaries}

In this section, we present some material needed for the statement and proof of our results. In what follows, we present some properties related to $W^{1, p(\cdot)}(\Omega)$ Sobolev spaces with variable exponents, see $[2,9,14]$. The spaces $L^{p(\cdot)}(\Omega)$ are special cases of the generalized Orlicz Spaces originated by Nakano [19] and developed by Musielak [17] and Orlicz [18]. The study of these spaces have been stimulated by problems of elasticity, fluid dynamics, calculus of variations and differential equations.

Let $p: \Omega \rightarrow[1, \infty)$ be a measurable function. We define the variable exponent Lebesgue space with variable exponent $p(\cdot)$ by:

$$
L^{p(\cdot)}(\Omega)=\left\{u: \Omega \rightarrow R ; \text { measurable in } \Omega: \int_{\Omega}|u|^{p(\cdot)} d x<\infty\right\},
$$

with a Luxemburg-type norm

$$
\|u\|_{p(\cdot)}=\inf \left\{\lambda>0: \int_{\Omega}\left|\frac{u}{\lambda}\right|^{p(x)} d x \leq 1\right\} .
$$

Equipped with this norm, $L^{p(\cdot)}(\Omega)$ is a Banach space (see [8]).

The relation between the modular $\int_{\Omega}|f|^{p(x)} d x$ and the norm follows from

$$
\min \left(\|f\|_{p(x)}^{p^{-}},\|f\|_{p(x)}^{p^{+}}\right) \leq \int_{\Omega}|f|^{p(x)} d x \leq \max \left(\|f\|_{p(x)}^{p^{-}},\|f\|_{p(x)}^{p^{+}}\right) .
$$

In the case $p(x)=$ const $>1$, these inequalities transform into equalities. For all $f \in L^{p(x)}(\Omega), g \in L^{p^{\prime}(x)}(\Omega)$ with

$$
p(x) \in(1, \infty), \quad p^{\prime}(x)=\frac{p(x)}{p(x)-1}
$$

the generalized Hölder inequality holds, that is,

Lemma 2.1. [1] (Hölder's inequality) Let $p, q, s \geq 1$ be measurable functions defined on $\Omega$ and

$$
\frac{1}{s(y)}=\frac{1}{p(y)}+\frac{1}{q(y)}, \text { for a.e. } y \in \Omega,
$$

satisfies. If $f \in L^{p(\cdot)}(\Omega)$ and $g \in L^{q(\cdot)}(\Omega)$, then, $f g \in L^{s(\cdot)}(\Omega)$ and

$$
\|f g\|_{s(\cdot)} \leq 2\|f\|_{p(\cdot)}\|g\|_{q(\cdot)} .
$$

Next, we define the variable-exponent Sobolev space $W^{1, p(\cdot)}(\Omega)$ as follows:

$$
W^{1, p(\cdot)}(\Omega)=\left\{u \in L^{p(\cdot)}(\Omega): \nabla u \text { exists and }|\nabla u| \in L^{p(\cdot)}(\Omega)\right\} .
$$

Variable exponent Sobolev space with respect to the norm:

$$
\|u\|_{1, p(\cdot)}=\|u\|_{p(\cdot)}+\|\nabla u\|_{p(\cdot)}
$$

is a Banach space. The space $W_{0}^{1, p(\cdot)}(\Omega)$ is defined as the closure of $C_{0}^{\infty}(\Omega)$ in $W^{1, p(\cdot)}(\Omega)$. For $u \in W_{0}^{1, p(\cdot)}(\Omega)$, we can define an equivalent norm:

$$
\|u\|_{1, p(\cdot)}=\|\nabla u\|_{p(\cdot)} .
$$

The dual of $W_{0}^{1, p(\cdot)}(\Omega)$ is defined as $W_{0}^{-1, p^{\prime}(\cdot)}(\Omega)$, similar to the usual Sobolev spaces, where $\frac{1}{p(\cdot)}+\frac{1}{p^{\prime}(\cdot)}=1$.

We also assume that:

$$
|m(x)-m(y)| \leq-\frac{B}{\log |x-y|} \text { for all } x, y \in \Omega,
$$

$B>0$ and $0<\delta<1$ with $|x-y|<\delta$. (log-Hölder condition)

Lemma 2.2. [2] (Poincare inequality) Suppose that $p(\cdot)$ satisfies (2.1) and let $\Omega$ be a bounded domain of $R^{n}$. Then,

$$
\|u\|_{p(\cdot)} \leq c\|\nabla u\|_{p(\cdot)} \text { for all } u \in W_{0}^{1, p(\cdot)}(\Omega),
$$

where $c=c\left(p^{-}, p^{+},|\Omega|\right)>0$.

Remark 2.3. We denote by c various positive constants which may be different at different occurrences. Also, throughout this paper, we use the embedding

$$
H_{0}^{2}(\Omega) \hookrightarrow H_{0}^{1}(\Omega) \hookrightarrow L^{p}(\Omega)
$$

which implies

$$
\|u\|_{p} \leq C\|\nabla u\| \leq C\|\Delta u\|
$$

where $2 \leq p<\infty(n=1,2), 2 \leq p \leq \frac{2 n}{n-2}(n \geq 3)$. 


\section{Stability of solutions}

In this section, we get the stability results for the problem (3.1)-(3.7), with the exponent $m(\cdot)$.

Similar to the work of [20], we introduce a new variable

$$
z(x, \rho, t)=u_{t}(x, t-\tau \rho), x \in \Omega, \rho \in(0,1), t>0 ;
$$

hence, we have

$$
\tau z_{t}(x, \rho, t)+z_{\rho}(x, \rho, t)=0, x \in \Omega, \rho \in(0,1), t>0 .
$$

Consequently, problem (1.1)- (1.5) is transformed to:

$$
\begin{array}{r}
u_{t t}+\Delta^{2} u-M\left(\|\nabla u\|^{2}\right) \Delta u+\mu_{1} u_{t}(x, t)\left|u_{t}(x, t)\right|^{m(x)-2}+\mu_{2} z(x, 1, t)|z(x, 1, t)|^{m(x)-2}=0, \text { in } \Omega \times(0, \infty), \\
\tau z_{t}(x, \rho, t)+z_{\rho}(x, \rho, t)=0 \text { in } \Omega \times(0,1) \times(0, \infty),
\end{array}
$$

with Dirichlet boundary condition

$$
\begin{array}{r}
u(x, t)=0 \text { on } \partial \Omega \times[0, \infty), \\
z(x, \rho, t)=0 \text { on } \partial \Omega \times[0,1) \times[0, \infty),
\end{array}
$$

and initial data

$$
\begin{array}{r}
u(x, 0)=u_{0}(x) \text { in } \Omega, \\
u_{t}(x, 0)=u_{1}(x) \text { in } \Omega, \\
z(x, \rho, 0)=f_{0}(x,-\rho \tau) \text { in } \Omega \times(0,1) .
\end{array}
$$

Similar to [16] we can define the strong solution as follows:

Definition 3.1. Fix $T>0$. We call $(u, z)$ a strong solution of (3.1)-(3.7) if

$$
\begin{array}{r}
u \in W^{2, \infty}\left([0, T) ; L^{2}(\Omega)\right) \cap W^{1, \infty}\left([0, T) ; H_{0}^{2}(\Omega)\right) \cap L^{\infty}\left([0, T) ; H^{2}(\Omega) \cap H_{0}^{2}(\Omega)\right), \\
\left.u_{t} \in L^{m(\dot{(}}(\Omega) \times[0, T)\right), \\
z \in W^{1, \infty}\left([0,1] \times[0, T) ; L^{2}(\Omega)\right) \cap L^{\infty}\left([0,1] ; L^{m(\dot{)}(\Omega) \cap[0, T)}\right),
\end{array}
$$

and $(u, z)$ satisfies the initial data and (3.1) in the following sense

$$
\begin{array}{r}
\int_{\Omega}\left[u_{t t}+\Delta^{2} u-M\left(\|\nabla u\|^{2}\right) \Delta u+\mu_{1} u_{t}(x, t)\left|u_{t}(x, t)\right|^{m(x)-2}+\mu_{2} z(x, 1, t)|z(x, 1, t)|^{m(x)-2}\right] v d x=0, \\
\int_{\Omega}\left[\tau_{z t}(x, \rho, t)+z_{\rho}(x, \rho, t)\right] w d x=0,
\end{array}
$$

for a.e. $t \in[0, t)$ and for $(v, w) \in H_{0}^{1}(\Omega) \cap L^{2}(\Omega)$.

In order to state our main result, we define the "modified" energy functional of (3.1) is given by

$$
E(t)=\frac{1}{2}\left\|u_{t}\right\|^{2}+\frac{1}{2}\|\Delta u\|^{2}+\frac{1}{2}\|\nabla u\|^{2}+\frac{1}{2(\gamma+1)}\|\nabla u\|^{2(\gamma+1)}+\int_{0}^{1} \int_{\Omega} \frac{\xi(x)|z(x, \rho, t)|^{m(x)}}{m(x)} d x d \rho,
$$

for $t \geq 0$, where $\xi$ is a continuous function yields

$$
\tau\left|\mu_{2}\right|(m(x)-1)<\xi(x)<\tau\left(\mu_{1} m(x)-\left|\mu_{2}\right|\right), x \in \bar{\Omega} .
$$

The following lemma gives that, $E(t)$ is decreasing under the condition $\mu_{1}>\left|\mu_{2}\right|$.

Lemma 3.2. Let $(u, z)$ be a solution of (3.1)-(3.7). Then, there exists some $C_{0}>0$ such that

$$
E^{\prime}(t) \leq-C_{0} \int_{\Omega}\left(\left|u_{t}\right|^{m(x)}+|z(x, 1, t)|^{m(x)}\right) d x \leq 0 .
$$

Proof. Multiplying (3.1) by $u_{t}$, integrating over $\Omega$, then, multiplying (3.2) by $\frac{1}{\tau} \xi(x)|z|^{m(x)-2} z$ and integrating over $\Omega \times(0,1)$, summing up, we obtain

$$
\begin{aligned}
& \frac{d}{d t}\left[\frac{1}{2}\left\|u_{t}\right\|^{2}+\frac{1}{2}\|\Delta u\|^{2}+\frac{1}{2}\|\nabla u\|^{2}+\frac{1}{2(\gamma+1)}\|\nabla u\|^{2(\gamma+1)}+\int_{0}^{1} \int_{\Omega} \frac{\xi(x)|z(x, \rho, t)|^{m(x)}}{m(x)} d x d \rho\right] \\
& =-\mu_{1} \int_{\Omega}\left|u_{t}\right|^{m(x)} d x-\frac{1}{\tau} \int_{\Omega} \int_{0}^{1} \xi(x)|z(x, \rho, t)|^{m(x)-2} z z_{\rho}(x, \rho, t) d \rho d x-\mu_{2} \int_{\Omega} u_{t} z(x, 1, t)|z(x, 1, t)|^{m(x)-2} d x .
\end{aligned}
$$


The last two terms of the right-hand side of (3.11) can be estimated as follows:

$$
\begin{aligned}
-\frac{1}{\tau} \int_{\Omega} \int_{0}^{1} \xi(x)|z(x, \rho, t)|^{m(x)-2} z z_{\rho}(x, \rho, t) d \rho d x & =-\frac{1}{\tau} \int_{\Omega} \int_{0}^{1} \frac{\partial}{\partial \rho}\left(\frac{\xi(x)|z(x, \rho, t)|^{m(x)}}{m(x)}\right) d \rho d x \\
& =\frac{1}{\tau} \int_{\Omega} \frac{\xi(x)}{m(x)}\left(|z(x, 0, t)|^{m(x)}-|z(x, 1, t)|^{m(x)}\right) d x \\
& =\int_{\Omega} \frac{\xi(x)}{\tau m(x)}\left|u_{t}\right|^{m(x)} d x-\int_{\Omega} \frac{\xi(x)}{\tau m(x)}|z(x, 1, t)|^{m(x)} .
\end{aligned}
$$

By using Young's inequality, $q=\frac{m(x)}{m(x)-1}$ and $q^{\prime}=m(x)$ for the last term, we get

$$
\left|u_{t}\right||z(x, 1, t)|^{m(x)-1} \leq \frac{1}{m(x)}\left|u_{t}\right|^{m(x)}+\frac{m(x)-1}{m(x)}|z(x, 1, t)|^{m(x)} .
$$

Consequently, we obtain

$$
-\mu_{2} \int_{\Omega} u_{t} z|z(x, 1, t)|^{m(x)-2} d x \leq\left|\mu_{2}\right|\left(\int_{\Omega} \frac{1}{m(x)}\left|u_{t}(t)\right|^{m(x)} d x+\int_{\Omega} \frac{m(x)-1}{m(x)}|z(x, 1, t)|^{m(x)} d x\right) .
$$

Thus,

$$
\frac{d E(t)}{d t} \leq-\int_{\Omega}\left[\mu_{1}-\left(\frac{\xi(x)}{\tau m(x)}+\frac{\left|\mu_{2}\right|}{m(x)}\right)\right]\left|u_{t}(t)\right|^{m(x)} d x-\int_{\Omega}\left(\frac{\xi(x)}{\tau m(x)}-\frac{\left|\mu_{2}\right|(m(x)-1)}{m(x)}\right)|z(x, 1, t)|^{m(x)} d x .
$$

As a result, for all $x \in \bar{\Omega}$, the relation (3.9) satisfies

$$
f_{1}(x)=\mu_{1}-\left(\frac{\xi(x)}{\tau m(x)}+\frac{\left|\mu_{2}\right|}{m(x)}\right)>0, \text { and } f_{2}(x)=\frac{\xi(x)}{\tau m(x)}-\frac{\left|\mu_{2}\right|(m(x)-1)}{m(x)}>0 .
$$

Since $m(x)$, and hence $\xi(x)$, is bounded, we infer that $f_{1}(x)$ and $f_{2}(x)$ are also bounded. Hence, if we define

$$
C_{0}(x)=\min \left\{f_{1}(x), f_{2}(x)\right\}>0 \text { for any } x \in \bar{\Omega},
$$

and take $C_{0}(x)=\inf _{\bar{\Omega}} C_{0}(x)$, so $C_{0}(x) \geq C_{0}>0$. Therefore,

$$
E^{\prime}(t) \leq-C_{0}\left[\int_{\Omega}\left|u_{t}(t)\right|^{m(x)} d x+\int_{\Omega}|z(x, 1, t)|^{m(x)} d x\right] \leq 0 .
$$

We need the following lemmas before obtain our stability results.

Lemma 3.3. (Komornik, [15]) Let $E: R^{+} \rightarrow R^{+}$be a nonincreasing function and suppose that there are constants $\sigma, \omega>0$ such that

$$
\int_{s}^{\infty} E^{1+\sigma}(t) d t \leq \frac{1}{\Omega} E^{\sigma}(0) E(s)=c E(s), \forall s>0 .
$$

Then, we have

$$
\left\{\begin{array}{l}
E(t) \leq c E(0)(1+t)^{1 / \sigma} \quad \text { if } \sigma>0, \\
E(t) \leq c E(0) e^{-\omega t} \quad \text { if } \sigma=0 .
\end{array}\right.
$$

for all $t \geq 0$.

Lemma 3.4. [16] The functional

$$
F(t)=\tau \int_{0}^{1} \int_{\Omega} e^{-\rho \tau \xi}(x)|z(x, \rho, t)|^{m(x)} d x d \rho
$$

satisfies

$$
F^{\prime}(t) \leq \int_{\Omega} \xi(x)\left|u_{t}\right|^{m(x)} d x-\tau e^{-\tau} \int_{0}^{1} \int_{\Omega} \xi(x)|z(x, \rho, t)|^{m(x)} d x d \rho
$$

along the solution of (3.1)-(3.7).

Theorem 3.5. Assume that conditions (1.6) and (2.1) are satisfied. Then, there exist two constants $c, \alpha>0$ independent of $t$ such that any global solution of (3.1)-(3.7) satisfies,

$$
\left\{\begin{array}{c}
E(t) \leq c e^{-\alpha t} \quad \text { if } m(\cdot)=2 \\
E(t) \leq c E(0)(1+t)^{2 /\left(m^{+}-2\right)} \text { if } m^{+}>2
\end{array}\right.
$$


Proof. We multiply the equation (3.1) by $u E^{q}(t)$, for $q>0$ to be specified later, and integrate over $\Omega \times(s, T), s<T$, to have

$$
\int_{s}^{T} E^{q}(t) \int_{\Omega}\left[u u_{t t}+u \Delta^{2} u-u \Delta u-\|\nabla u\|^{2 \gamma} u \Delta u+\mu_{1} u u_{t}\left|u_{t}\right|^{m(x)-2}+\mu_{2} u z(x, 1, t)|z(x, 1, t)|^{m(x)-2}\right] d x d t=0,
$$

which implies that

$$
\int_{S}^{T} E^{q}(t) \int_{\Omega}\left(\frac{d}{d t}\left(u u_{t}\right)-u_{t}^{2}+|\Delta u|^{2}+|\nabla u|^{2}+\|\nabla u\|^{2 \gamma}|\nabla u|^{2}+\mu_{1} u u_{t}(x, t)\left|u_{t}(x, t)\right|^{m(x)-2}+\mu_{2} u z(x, 1, t)|z(x, 1, t)|^{m(x)-2}\right) d x d t=0 .
$$

Recalling the definition of $E(t)$, given in (3.8) adding and subtracting some terms and using the relation

$$
\frac{d}{d t}\left(E^{q}(t) \int_{\Omega} u u_{t} d x\right)=q E^{q-1}(t) E^{\prime}(t) \int_{\Omega} u u_{t} d x+E^{q}(t) \frac{d}{d t} \int_{\Omega} u u_{t} d x
$$

the equation (3.12) satisfies

$$
\begin{aligned}
2 \int_{s}^{T} E^{q+1}(t) d t & =-\int_{s}^{T} \frac{d}{d t}\left(E^{q}(t) \int_{\Omega} u u_{t} d x\right) d t+q \int_{s}^{T} E^{q-1}(t) E^{\prime}(t) \int_{\Omega} u u_{t} d x d t \\
& -\frac{\gamma}{\gamma+1} \int_{S}^{T} E^{q} \int_{\Omega}\|\nabla u\|^{2 \gamma}\left|\nabla u^{2}\right| d x d t+2 \int_{s}^{T} E^{q}(t) \int_{\Omega} u_{t}^{2} d x d t-\mu_{1} \int_{s}^{T} E^{q}(t) \int_{\Omega} u u_{t}\left|u_{t}\right|^{m(x)-2} d x d t \\
& -\mu_{2} \int_{s}^{T} E^{q}(t) \int_{\Omega} u z(x, 1, t)|z(x, 1, t)|^{m(x)-2} d x d t+2 \int_{s}^{T} E^{q}(t) \int_{0}^{1} \int_{\Omega} \frac{\xi(x)|z(x, \rho, t)|^{m(x)}}{m(x)} d x d \rho d t
\end{aligned}
$$

Next, we estimate the parts of the right side in inequality (3.13), respectively.

The first term is estimated as follows:

$$
\begin{aligned}
\left|-\int_{s}^{T} \frac{d}{d t}\left(E^{q}(t) \int_{\Omega} u u_{t} d x\right) d t\right| & =\left|E^{q}(s) \int_{\Omega} u u_{t}(x, s) d x-E^{q}(T) \int_{\Omega} u u_{t}(x, T) d x\right| \\
& \leq \frac{1}{2} E^{q}(s)\left[\int_{\Omega} u^{2}(x, s) d x+\int_{\Omega} u_{t}^{2}(x, s) d x\right]+\frac{1}{2} E^{q}(T)\left[\int_{\Omega} u^{2}(x, T) d x+\int_{\Omega} u_{t}^{2}(x, T) d x\right] \\
& \leq \frac{1}{2} E^{q}(s)\left[C_{p}\|\Delta u(s)\|_{2}^{2}+2 E(s)\right]+\frac{1}{2} E^{q}(T)\left[C_{p}\|\Delta u(T)\|_{2}^{2}+2 E(T)\right] \\
& \leq E^{q}(s)\left[C_{p} E(s)+E(s)\right]+E^{q}(T)\left[C_{p} E(T)+E(T)\right]
\end{aligned}
$$

where $C_{p}$ is the Poincare's constant. Because of $E(t)$ is nonincreasing, we infer that

$$
\left|-\int_{s}^{T} \frac{d}{d t}\left(E^{q}(t) \int_{\Omega} u u_{t} d x\right) d t\right| \leq c E^{q+1}(s) \leq c E^{q}(0) E(s) \leq c E(s) .
$$

In similar way, we handle the term

$$
\begin{aligned}
\left|q \int_{s}^{T} E^{q-1}(t) E^{\prime}(t) \int_{\Omega} u u_{t} d x d t\right| & \leq-q \int_{s}^{T} E^{q-1}(t) E^{\prime}(t)\left[C_{p} E(T)+E(T)\right] d t \\
& \leq-c \int_{s}^{T} E^{q}(t) E^{\prime}(t) \leq c E^{q+1}(s) \leq c E(s) .
\end{aligned}
$$

We estimate the other term as follows:

$$
\begin{aligned}
\left|-\frac{\gamma}{\gamma+1} \int_{s}^{T} E^{q} \int_{\Omega}\|\nabla u\|^{2 \gamma}\right| \nabla u^{2}|d x d t| & =\left|-2 \gamma \int_{s}^{T} E^{q}\left(\frac{\|\nabla u\|^{2 \gamma}}{2(\gamma+1)} \int_{\Omega}\left|\nabla u^{2}\right| d x\right) d t\right| \\
& =\left|-2 \gamma \int_{s}^{T} E^{q}\left(\frac{\|\nabla u\|^{2(\gamma+1)}}{2(\gamma+1)}\right) d t\right| \\
& \leq\left|-2 \gamma \int_{s}^{T} E^{q}(E(t)) d t\right| \\
& \leq C^{*} \int_{s}^{T} E^{q+1}(t) d t \\
& \leq C^{*} E(s)
\end{aligned}
$$

where $C^{*}$ is a generic constant.

To treat the other term, we set

$\Omega_{+}=\left\{x \in \Omega,\left|u_{t}(x, t)\right| \geq 1\right\}$ and $\Omega_{-}=\left\{x \in \Omega,\left|u_{t}(x, t)\right|<1\right\}$. 
Then, by using the Hölder's and Young's inequalities, we get

$$
\begin{aligned}
\left|\int_{s}^{T} E^{q}(t) \int_{\Omega} u_{t}^{2} d x d t\right| & =\left|\int_{s}^{T} E^{q}(t)\left[\int_{\Omega_{+}} u_{t}^{2} d x+\int_{\Omega_{-}} u_{t}^{2} d x\right] d t\right| \\
& \leq c \int_{s}^{T} E^{q}(t)\left[\left(\int_{\Omega_{+}}\left|u_{t}\right|^{m^{-}} d x\right)^{2 / m^{-}}+\left(\int_{\Omega_{-}}\left|u_{t}\right|^{m^{+}} d x\right)^{2 / m^{+}}\right] d t \\
& \leq c \int_{s}^{T} E^{q}(t)\left[\left(\int_{\Omega}\left|u_{t}\right|^{m(x)} d x\right)^{2 / m^{-}}+\left(\int_{\Omega}\left|u_{t}\right|^{m(x)} d x\right)^{2 / m^{+}}\right] d t \\
& \leq c \int_{s}^{T} E^{q}(t)\left[\left(-E^{\prime}(t)\right)^{2 / m^{-}}+\left(-E^{\prime}(t)\right)^{2 / m^{+}}\right] d t \\
& \leq c \varepsilon \int_{s}^{T}[E(t)]^{q m^{-} /\left(m^{-}-2\right)} d t+c(\varepsilon) \int_{s}^{T}\left(-E^{\prime}(t)\right) d t+c \varepsilon \int_{s}^{T} E(t)^{q+1} d t+c(\varepsilon) \int_{s}^{T}\left(-E^{\prime}(t)\right)^{2(q+1) / m^{+}} d t
\end{aligned}
$$

For $m^{-}>2$ and the choice of $q=m^{+} / 2-1$ will give $\frac{q m^{-}}{m^{-}-2}=q+1+\frac{m^{+}-m^{-}}{m^{-}-2}$.

Therefore,

$$
\begin{aligned}
\left|\int_{s}^{T} E^{q}(t) \int_{\Omega} u_{t}^{2} d x d t\right| & \leq c \varepsilon \int_{s}^{T} E(t)^{q+1} d t+c \varepsilon[E(0)]^{\frac{m^{+}-m^{-}}{m^{-}-2}} \int_{s}^{T}[E(t)]^{q+1} d t+c(\varepsilon) E(s) \\
& \leq c \varepsilon \int_{s}^{T} E(t)^{q+1} d t+c(\varepsilon) E(s) .
\end{aligned}
$$

For the case $m^{-}=2$ and the choice of $q=m^{+} / 2-1$ will give the similar result.

For the other term, utilizing Young's inequality we conclude

$$
\begin{aligned}
\left.\left|-\mu_{1} \int_{s}^{T} E^{q}(t) \int_{\Omega} u\right| u_{t}\right|^{m(x)-1} d x d t \mid & \leq \varepsilon \int_{s}^{T} E^{q}(t) \int_{\Omega}|u(t)|^{m(x)} d x d t+c \int_{s}^{T} E^{q}(t) \int_{\Omega} c_{\varepsilon}(x)\left|u_{t}(t)\right|^{m(x)} d x d t \\
& \leq \varepsilon \int_{s}^{T} E^{q}(t)\left[\int_{\Omega_{+}}|u(t)|^{m^{-}} d x+\int_{\Omega_{-}}|u(t)|^{m^{+}} d x\right] d t+c \int_{s}^{T} E^{q}(t) \int_{\Omega} c_{\varepsilon}(x)\left|u_{t}(t)\right|^{m(x)} d x d t,
\end{aligned}
$$

where we have used Young's inequality with

$$
p(x)=\frac{m(x)}{m(x)-1}, p^{\prime}(x)=m(x)
$$

and hence

$$
c_{\mathcal{E}}(x)=(m(x)-1) m(x)^{m(x) /(1-m(x))} \varepsilon^{1 /(1-m(x))} .
$$

By using the embeddings $H_{0}^{2}(\Omega) \hookrightarrow L^{m^{-}}(\Omega)$ and $H_{0}^{2}(\Omega) \hookrightarrow L^{m^{+}}(\Omega)$, we obtain

$$
\begin{aligned}
\left.\left|-\mu_{1} \int_{s}^{T} E^{q}(t) \int_{\Omega} u\right| u_{t}\right|^{m(x)-1} d x d t \mid & \leq \varepsilon \int_{s}^{T} E^{q}(t)\left[c\|\Delta u(s)\|_{2}^{m^{-}}+c\|\Delta u(s)\|_{2}^{m^{+}}\right] d t+c \int_{s}^{T} E^{q}(t) \int_{\Omega} c_{\varepsilon}(x)\left|u_{t}(t)\right|^{m(x)} d x d t \\
& \leq \varepsilon \int_{s}^{T} E^{q}(t)\left[c E^{\left(m^{-}-2\right) / 2}(0) E(t)+c E^{\left(m^{+}-2\right) / 2}(0) E(t)\right] d t+c \int_{s}^{T} E^{q}(t) \int_{\Omega} c_{\varepsilon}(x)\left|u_{t}(t)\right|^{m(x)} d x d t \\
& \leq c \varepsilon \int_{s}^{T} E^{q+1}(t) d t+\int_{s}^{T} E^{q}(t) \int_{\Omega} c_{\varepsilon}(x)\left|u_{t}(t)\right|^{m(x)} d x d t
\end{aligned}
$$

The next term of (3.13) can be estimated in a similar attitude to get

$$
\begin{aligned}
\left.\left|-\mu_{2} \int_{s}^{T} E^{q}(t) \int_{\Omega} u\right| z(x, 1, t)\right|^{m(x)-1} d x d t \mid & \leq \varepsilon \int_{s}^{T} E^{q}(t)\left[c\|\Delta u(s)\|_{2}^{m^{-}}+c\|\Delta u(s)\|_{2}^{m^{+}}\right] d t+c \int_{s}^{T} E^{q}(t) \int_{\Omega} c_{\varepsilon}(x)|z(x, 1, t)|^{m(x)} d x d t \\
& \leq c \varepsilon \int_{s}^{T} E^{q+1}(t) d t+\int_{s}^{T} E^{q}(t) \int_{\Omega} c_{\varepsilon}(x)|z(x, 1, t)|^{m(x)} d x d t
\end{aligned}
$$

For the last term of (3.13), from Lemma 3.4, we get

$$
\begin{aligned}
2 \int_{s}^{T} E^{q}(t) \int_{0}^{1} \int_{\Omega} \frac{\xi(x)|z(x, \rho, t)|^{m(x)}}{m(x)} d x d \rho d t & \leq \frac{2}{m^{-}} \int_{s}^{T} E^{q}(t) \int_{0}^{1} \int_{\Omega} \xi(x)|z(x, \rho, t)|^{m(x)} d x d \rho d t \\
& \leq-\frac{2 \tau}{m^{-}} \int_{s}^{T} E^{q}(t) \frac{d}{d t}\left(\int_{0}^{1} \int_{\Omega} e^{-\rho \tau \xi} \xi(x)|z|^{m(x)} d x d \rho\right) d t \\
& +\frac{2}{m^{-}} \int_{s}^{T} E^{q}(t) \int_{\Omega} \xi(x)\left|u_{t}\right|^{m(x)} d x d t \\
& \leq-\frac{2 \tau}{m^{-}}\left[E^{q}(t) \int_{0}^{1} \int_{\Omega} e^{-\rho \tau} \xi(x)|z|^{m(x)} d x d \rho\right]_{t=s}^{t=T}+\frac{2}{m^{-}} \int_{s}^{T} E^{q}(t) \int_{\Omega} \xi(x)\left|u_{t}\right|^{m(x)} d x d t
\end{aligned}
$$


As $\xi(x)$ is bounded, by (3.8), we obtain

$$
\begin{aligned}
2 \int_{s}^{T} E^{q}(t) \int_{0}^{1} \int_{\Omega} \frac{\xi(x)|z(x, \rho, t)|^{m(x)}}{m(x)} d x d \rho d t & \leq \frac{2 \tau e^{-\tau}}{m^{-}} E^{q}(s) E(s)+\frac{2 c}{m^{-}} E^{q+1}(T) \\
& \leq \frac{2 \tau e^{-\tau}}{m^{-}} E^{q}(0) E(s)+\frac{2 c}{m^{-}} E^{q}(T) E(s) \leq c E(s),
\end{aligned}
$$

for some $c>0$.

By combining (3.13)-(3.20), we conclude that

$$
\int_{s}^{T} E^{q+1}(t) d t \leq \varepsilon \int_{s}^{T} E^{q+1}(t) d t+c E(s)+c \int_{s}^{T} E^{q}(t) \int_{\Omega} c_{\varepsilon}(x)|z(x, 1, t)|^{m(x)} d x d t .
$$

Choosing $\varepsilon$ so small such that

$$
\int_{s}^{T} E^{q+1}(t) d t \leq c E(s)+c \int_{s}^{T} E^{q}(t) \int_{\Omega} c_{\mathcal{E}}(x)|z(x, 1, t)|^{m(x)} d x d t .
$$

Once $\varepsilon$ is fixed, then $c_{\varepsilon}(x) \leq M$, since $m(x)$ is bounded. Therefore, we infer that

$$
\begin{aligned}
\int_{s}^{T} E^{q+1}(t) d t & \leq c E(s)+c M \int_{s}^{T} E^{q}(t) \int_{\Omega}|z(x, 1, t)|^{m(x)} d x d t \\
& \leq c E(s)-C_{0} M \int_{s}^{T} E^{q}(t) E^{\prime}(t) d t \\
& \leq c E(s)+\frac{C_{0} M}{q+1}\left[E^{q+1}(s)-E^{q+1}(T)\right] \leq c E(s) .
\end{aligned}
$$

By taking $T \rightarrow \infty$, we obtain

$$
\int_{s}^{\infty} E^{q+1}(t) d t \leq c E(s)
$$

Thus, Komornik's Lemma (with $\sigma=q=m^{+} / 2-1$ ) implies the desired result.

\section{Acknowledgements}

The authors would like to express their sincere thanks to the editor and the anonymous reviewers for their helpful comments and suggestions.

\section{Funding}

There is no funding for this work.

\section{Availability of data and materials}

Not applicable.

\section{Competing interests}

The authors declare that they have no competing interests.

\section{Author's contributions}

All authors contributed equally to the writing of this paper. All authors read and approved the final manuscript.

\section{References}

[1] S. Antontsev, Wave equation with $p(x, t)$-Laplacian and damping term: blow-up of solutions, C. R. Mecanique, 339 (2011), $751-755$.

[2] S. Antontsev, Wave equation with $p(x, t)$-Laplacian and damping term: existence and blow-up, Differential Equations Appl., 3 (2011), $503-525$.

[3] S. Antontsev, J. Ferreira, E. Pişkin, Existence and blow up of solutions for a strongly damped Petrovsky equation with variable-exponents nonlinearities, Electr. j. differ. equ., 2021(6) (2021), 1-18

[4] S. Antontsev, J. Ferreira, E. Pişkin, S. M. Siqueira Cordeiro, Existence and non-existence of solutions for Timoshenko-type equations with variable exponents, Nonlinear Anal. Real World Appl., 61 (2021), Article ID 103341.

[5] A. Antontsev, J. Ferreira, E. Pişkin, H. Yüksekkaya, M. Shahrouzi, Blow up and asymptotic behavior of solutions for a p(x)-Laplacian equation with delay term and variable exponents, Electron. J. Differ. Equ., 2021(84), (2021), 1-20.

[6] J. M. Ball, Initial boundary value problem for an extensible beam, J. Math. Anal. Appl., 42 (1973), 61-90.

[7] Y. Chen, S. Levine, M. Rao, Variable exponent, linear growth functionals in image restoration, SIAM J. Appl. Math., 66 (2006), $1383-1406$.

[8] L. Diening, P. Hasto, P. Harjulehto, M. M. Ruzicka, Lebesgue and Sobolev Spaces with Variable Exponents, Springer-Verlag, 2011.

[9] X. L. Fan, J. S. Shen, D. Zhao, Sobolev embedding theorems for spaces $W^{k, p(x)}(\Omega)$, J. Math. Anal. Appl., 263 (2001), $749-760$.

[10] B. Feng, H. Li, Energy decay for a viscoelastic Kirchhoff plate equation with a delay term, Bound. Value Probl., 174 (2016), https://doi.org/10.1186/s13661-016-0682-8

[11] J. R. Kang, Global nonexistence of solutions for von Karman equations with variable exponents, Appl. Math. Lett., 86 (2018), $249-255$.

[12] M. Kafini, S. A. Messaoudi, A blow-up result in a nonlinear wave equation with delay, Mediterr. J. Math., 13 (2016), $237-247$.

[13] G. Kirchhoff, Vorlesungen über mechanik, B. G. Teubner, Leipzig, 1897. 
[14] O. Kovacik, J. Rakosnik, On spaces $L^{p(x)}(\Omega)$, and $W^{k, p(x)}(\Omega)$, Czech. Math. J., 41(116) (1991), 592-618.

[15] V. Komornik, Exact Controllability and Stabilization. The Multiplier Method, Masson and Wiley, 1994.

[16] S. A. Messaoudi, M. Kafini, On the decay and global nonexistence of solutions to a damped wave equation with variable-exponent nonlinearity and delay, Ann. Pol. Math., 122 (2019), 49-70.

[17] J. Musielak, Orlicz spaces and modular spaces, Springer-Verlag, Berlin, 1983.

[18] J. Musielak, W. Orlicz, On modular spaces, Studia Math. 18, (1959) 49-65.

[19] H. Nakano, Modulared semi-ordered linear spaces, Maruzen Co., Tokyo, 1950.

[20] S. Nicaise, C. Pignotti, Stability and instability results of the wave equation with a delay term in the boundary or internal feedbacks, SIAM J. Control Optim., 45 (2006), 1561-1585.

[21] S. H. Park, J. R. Kang, Blow-up of solutions for a viscoelastic wave equation with variable exponents, Math. Meth. Appl. Sci., 42 (2019), 2083-2097.

22] E. Piskin, Finite time blow up of solutions of the Kirchhoff-type equation with variable exponents, Int. J. Nonlinear Anal. Appl., 11(1) (2020), 37-45.

[23] E. Pişkin, H. Yüksekkaya, Decay of solutions for a nonlinear Petrovsky equation with delay term and variable exponents, The Aligarh Bull. of Maths., 39(2) (2020), 63-78.

[24] H. Yüksekkaya, E. Pişkin, S.M. Boulaaras, B. B. Cherif, Existence, decay and blow-up of solutions for a higher-order kirchhoff-type equation with delay term, J. Funct. Spaces, 2021 (2021), Article ID 4414545

[25] H. Yüksekkaya, E. Pişkin, S. M. Boulaaras, B. B. Cherif, S. A. Zubair, Existence, nonexistence, and stability of solutions for a delayed plate equation with the logarithmic source, Adv. Math. Phys., 2021 (2021), 1-11.

[26] M. Ruzicka, Electrorheological Fluids: Modeling and Mathematical Theory, Lecture Notes in Mathematics, Springer, 2000.

[27] M. L. Santos, J. Ferreira, C. A. Raposo, Existence and uniform decay for a nonlinear beam equation with nonlinearity of Kirchhoff type in domains with moving boundary, Abstr. Appl. Anal., 8 (2005), 901-919.

[28] M. Shahrouzi, On behaviour of solutions for a nonlinear viscoelastic equation with variable-exponent nonlinearities, Comput. Math. with Appl., 75(11) (2018), 3946-3956.

[29] M. Tucsnak, Semi-internal stabilization for a nonlinear Euler-Bernoulli equation, Math. Method. Appl. Sci., 19 (1996), 897-907.

[30] S. Woinowsky-Krieger, The effect of axial force on the vibration of hinged bars, J. Appl. Mech., 17 (1950), 35-36. 